\title{
Age and Incidence of Dementia Diagnosis
}

J Gen Intern Med 36(7):2167-9

DOI: $10.1007 / \mathrm{s} 11606-020-05895-y$

(c) Society of General Internal Medicine 2020

\section{INTRODUCTION}

Growth in the US population of older adults places an increasing number of people at risk of developing dementia. Most, but not all, studies report an increase in dementia incidence with each successive age group. ${ }^{1}$ Results have also been inconsistent regarding whether incidence of dementia varies between men and women of the same age. Although the incidence of dementia is higher for similarly aged black than white adults, ${ }^{2}$ whether age-adjusted incidence differs between other racial/ethnic groups remains unclear. ${ }^{3}$ Prior epidemiological studies on age of incidence of dementia have been hindered by relatively small in-person assessed samples ${ }^{4}$ or larger claims-based cohorts that are not nationally representative. ${ }^{3}$ The objective of this analysis was to estimate the incidence of clinical dementia diagnoses by single year of age, overall and stratified by gender and race/ethnicity by examining clinical administrative claims from a large national Medicare population.

\section{METHODS}

Administrative data from a 50\% random national sample of Medicare beneficiaries aged 65-100 years with fee-for-service and Part D prescription drug coverage (2007-2017) were used to identify incident dementia diagnoses $(\geq 2$ outpatient or $\geq 1$ inpatient) among persons 66 to 100 years of age. Incident dementia was defined by a first observed qualifying code from the CMS Chronic Condition Warehouse algorithm for Alzheimer's disease and related disorders or senile dementia following $\geq 12$ consecutive months of Medicare eligibility without a qualifying code. As compared with in-person assessments of cognitive impairment, this claims-based definition has a sensitivity of $85.5 \%$, specificity of $85.9 \%$, and kappa of $0.70 .^{5}$ Incident dementia diagnoses were plotted by single year of age for the overall sample and separately by gender and major racial/ethnic groups. The Institutional Review Board of Rutgers University approved this study with a waiver of informed consent.

Received April 7, 2020

Accepted April 30, 2020

Published online June 3, 2020

\section{RESULTS}

Among 8,106,150 adult Medicare beneficiaries contributing $40,250,286$ years of follow-up, the annual incidence of dementia diagnoses per 1000 person-years increased from 7.6 (95\%CI 7.5-7.7) for adults aged 66 years to 159.6 (95\% CI 155.7-163.5) for adults 100 years of age. For men, the increase in incidence per 1000 person-years was from 8.3 (95\%CI 8.1-8.4) for adults aged 66 years to 142.4 (95\% CI 132.7-152.1) for adults aged 100 years and for women the increase was from $7.2(95 \% \mathrm{CI} 7.1-7.2)$ to 162.7 (95\%CI 158.5-167.0) per 1000 person-years (Fig. 1). The annual incidence of dementia diagnosis per 1000 person-years increased among adults aged 66 to 100 years for Asian/Pacific Islanders from 6.0 (95\%CI 5.5-6.4) to 142.1 (95\%CI $118.3-$ 166.0), for non-Hispanic whites from 6.7 (95\% CI 6.6-6.7) to 156.5 (95\% CI 152.3-160.7), for Hispanics from 11.1 (95\%CI $10.7-11.5)$ to 191.6 (95\%CI $168.9-214.4)$, and for nonHispanic blacks from 16.3 (95\%CI $16.0-16.7$ ) to 180.7 (95\%CI 166.1-195.3) (Fig. 2).

\section{DISCUSSION}

The risk of receiving a clinical dementia diagnosis increased with each successive year of life until age 98. As compared with women, men had a higher incidence of dementia diagnosis before age 70 and a lower incidence thereafter. A greater risk among women starting in the eighth decade of life might be related to selective survival of men with healthier cardiovascular risk profiles, sex differences in educational attainment, sex hormone exposures, or other factors. Consistent with prior research, non-Hispanic black adults had a higher incidence of dementia than non-Hispanic white adults, ${ }^{2}$ a difference that might be related to variation in vascular health or socioeconomic risk factors. A higher incidence of dementia diagnoses among Hispanic than non-Hispanic white or Asian/ Pacific Islander adults highlights the need for research aimed at discovering determinants of these differences including contributions of genetic factors, such as the apolipoprotein E $\varepsilon 4$ allele, environmental factors, and gene by environment interactions.

Limitations include that most but not all older US adults enroll in fee-for-service Medicare with prescription coverage; adults first diagnosed prior to age 66 years were not included in this analysis; heterogeneity within racial/ethnic groups; some older adults with dementia may not receive timely diagnoses; and potential misclassification of dementia status due to differences in access to specialty care across gender and racial/ethnic groups. Cultural differences in expectations of 


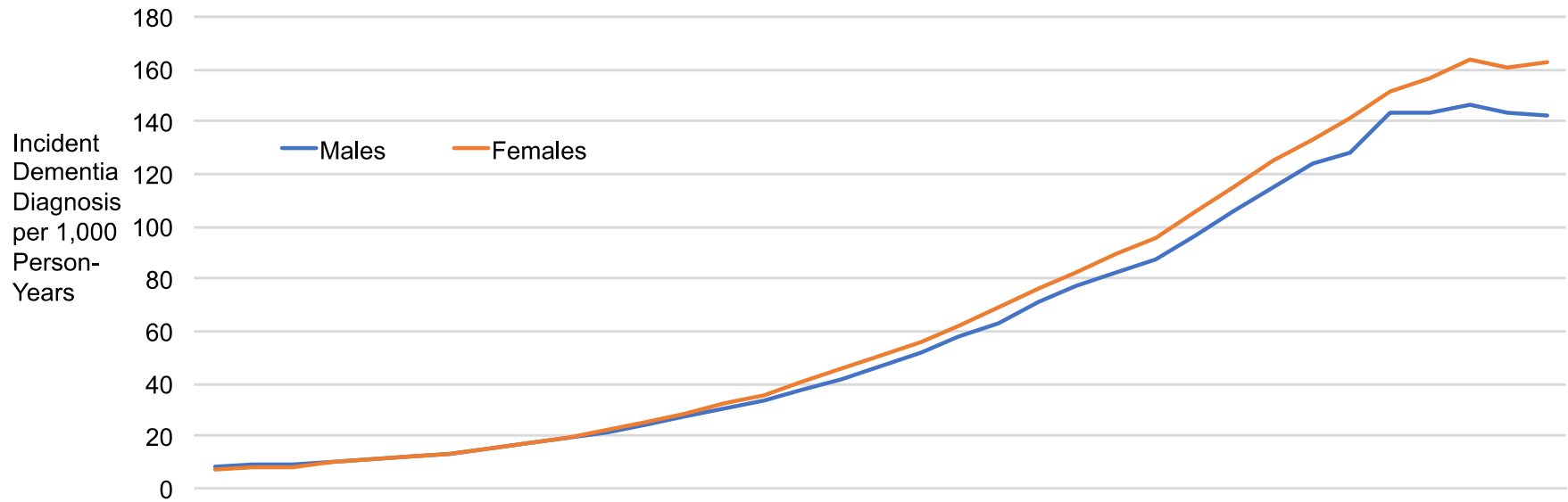

66676869707172737475767778798081828384858687888990919293949596979899100

Age in Years

Figure 1 Age-specific incidence rates of dementia diagnoses per 1000 person-years for males and females. Data from the Centers for Medicare and Medicaid Services. Incident dementia diagnoses per 1000 person-years with 95\% confidence intervals were for males aged 66 (8.3, 8.1-8.4), $80(33.7,33.4-34.1), 90(87.7,86.5-89.4)$, and $100(142.4,132.7-152.1)$ years and for females aged $66(7.2,7.1-7.2), 80(35.6,35.3-35.9), 90(95.7$, 94.9-96.4) and $100(162.7,158.5-167.0)$ years.

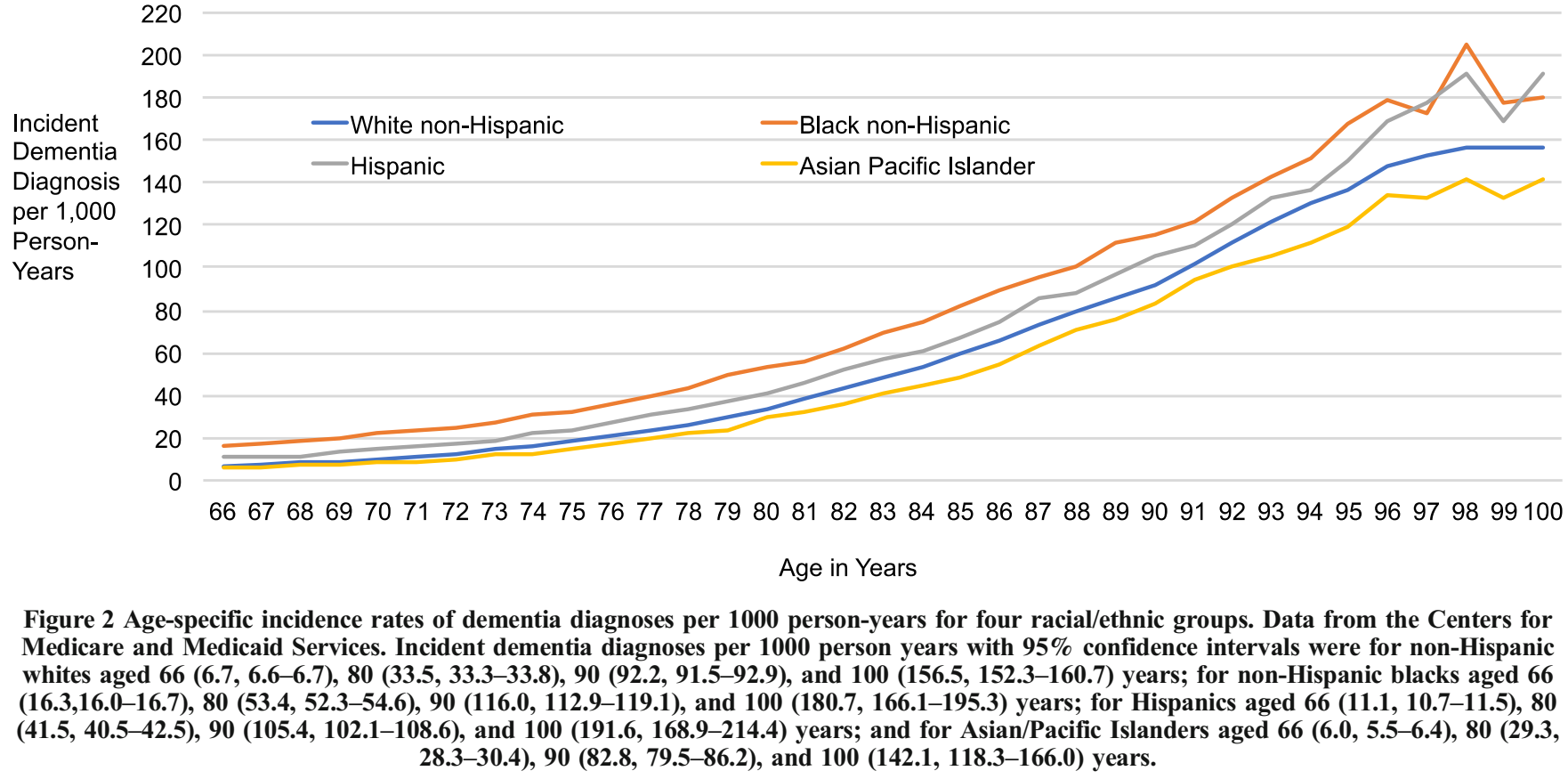

healthy aging may also contribute to racial/ethnic and gender differences in the clinical diagnosis of dementia.

Age-specific incidence of clinical dementia diagnosis varies by gender and race/ethnicity. Beyond estimating the clinical and societal burden of dementia, Medicare claims data might also inform the evaluation of clinical and public health initiatives to reduce the incidence of dementia.

Mark Olfson, $M D, M P H^{1}$

Thomas Scott Stroup, MD, MPH ${ }^{1}$

Cecilia Huang, $P h D^{2}$

Melanie M. Wall, $P h D^{1}$

Tobias Gerhard, $P h D^{2,3}$
${ }^{1}$ Department of Psychiatry, New York State Psychiatric Institute, Vagelos College of Physicians and Surgeons, Columbia University Irving Medical Center

1051 Riverside Drive, New York, NY 10032, USA

${ }^{2}$ Institute for Health, Health Care Policy and Aging Research, Rutgers University,

112 Paterson Street, New Brunswick, NJ 08901, USA

${ }^{3}$ Ernest Mario School of Pharmacy, Rutgers University,

160 Frelinghuysen Road, Piscataway, NJ 08854, USA

Corresponding Author: Mark Olfson, MD, MPH; Department of Psychiatry, New York State Psychiatric Institute, Vagelos College of Physicians and Surgeons, Columbia University Irving Medical Center 
1051 Riverside Drive, New York, NY 10032, USA (e-mail: Mark.Olfson@nyspi.columbia.edu).

Funding Information This work is supported by NIMH P5O MH115843 (Dr. Stroup).

\section{Compliance with Ethical Standards:}

The Institutional Review Board of Rutgers University approved this study with a waiver of informed consent.

Conflict of Interest: The authors declare that they do not have a conflict of interest.

\section{REFERENCES}

1. Tom SE, Hubbard RA, Crane PK, Haneuse SJ, et al., Characterization of dementia and Alzheimer's Disease in an older population: updated incidence and life expectancy with and without dementia. Am J Pub Health 2015; 105:408-413.

2. Steenland K, Goldstein F, Levey A, Wharton W. A meta-analysis of Alzheimer's Disease incidence and prevalence comparing AfricanAmericans and Caucasians. J Alzheimers Dis 2016;50:71-76.

3. Mayeda ER, Glymour MM, Qunesenberry CP, Whitmer RA. Inequalities in dementia incidence between six racial and ethnic groups over 14 years. Alzheimers Dement 2016(12):216-224.

4. Plassman BL, Langa KM, Fisher GG, Heeringa SG, et al. Incidence of dementia and cognitive impairment, not dementia in the United States. Ann Neurol 2011;70:418-426.

5. Taylor DH, Ostbye T, Langa K, Weir D, et al., The accuracy of Medicare claims as an epidemiological tool: the case of dementia revisited. $J$ Alzheimers Dis 2009;17:807-815.

Publisher's Note: Springer Nature remains neutral with regard to jurisdictional claims in published maps and institutional affiliations. 